\title{
Targeted Molecular Radiotherapy of Pediatric Solid Tumors Using a Radioiodinated Alkyl-Phospholipid Ether Analog
}

\author{
Dana C. Baiu ${ }^{1}$, Ian R. Marsh ${ }^{2}$, Alexander E. Boruch ${ }^{1}$, Ankita Shahi ${ }^{1}$, Saswati Bhattacharya ${ }^{1}$, Justin J. Jeffery ${ }^{3}$, \\ Qianqian Zhao ${ }^{4}$, Lance T. Hall ${ }^{3}$, Jamey P. Weichert ${ }^{2,3}$, Bryan P. Bednarz ${ }^{2}$, and Mario Otto ${ }^{1}$ \\ ${ }^{1}$ Department of Pediatrics, Carbone Cancer Center, School of Medicine and Public Health, University of Wisconsin-Madison, \\ Madison, Wisconsin; ${ }^{2}$ Department of Medical Physics, School of Medicine and Public Health, University of Wisconsin-Madison, \\ Madison, Wisconsin; ${ }^{3}$ Department of Radiology, School of Medicine and Public Health, University of Wisconsin-Madison, Madison, \\ Wisconsin; and ${ }^{4}$ Department of Biostatistics and Medical Informatics, School of Medicine and Public Health, University of \\ Wisconsin-Madison, Madison, Wisconsin
}

External-beam radiotherapy plays a critical role in the treatment of most pediatric solid tumors. Particularly in children, achieving an optimal therapeutic index to avoid damage to normal tissue is extremely important. Consequently, in metastatic disease, the utility of external-beam radiotherapy is limited. Molecular radiotherapy with tumor-targeted radionuclides may overcome some of these challenges, but to date there exists no single cancer-selective agent capable of treating various pediatric malignancies independently of their histopathologic origin. We tested the therapeutic potential of the clinical-grade alkyl-phospholipid ether analog CLR1404, 18-(piodophenyl)octadecyl phosphocholine, as a scaffold for tumortargeted radiotherapy of pediatric malignancies. Methods: Uptake of CLR1404 by pediatric solid tumor cells was tested in vitro by flow cytometry and in vivo by PET/CT imaging and dosimetry. The therapeutic potential of ${ }^{131}$ I-CLR1404 was evaluated in xenograft models. Results: In vitro, fluorescent CLR1404-BODIPY showed significant selective uptake in a variety of pediatric cancer lines compared with normal controls. In vivo tumor-targeted uptake in mouse xenograft models using ${ }^{124}$ I-CLR1404 was confirmed by imaging. Single-dose intravenous injection of ${ }^{131}$ I-CLR1404 significantly delayed tumor growth in all rodent pediatric xenograft models and extended animal survival while demonstrating a favorable side effect profile. Conclusion: ${ }^{131}$ I-CLR1404 has the potential to become a tumor-targeted radiotherapeutic drug with broad applicability in pediatric oncology. Because ${ }^{131}$ I-CLR1404 has entered clinical trials in adults, our data warrant the development of pediatric clinical trials for this particularly vulnerable patient population.

Key Words: targeted radiotherapy; pediatric cancer; radionuclide therapy; microPET/CT imaging

J Nucl Med 2018; 59:244-250

DOI: 10.2967/jnumed.117.193748

$\mathbf{E}$ xternal-beam radiation therapy plays an indispensable role in both curative and palliative treatment approaches for managing pediatric solid cancers $(1,2)$. Although most pediatric solid cancers are radiosensitive, high doses or large areas of exposure to radiation

Received Mar. 22, 2017; revision accepted Jun. 6, 2017.

For correspondence or reprints contact: Mario Otto, Department of Pediatrics, WIMR 4153, 1111 Highland Ave., Madison WI 53705.

E-mail: motto@pediatrics.wisc.edu

Published online Jul. 26, 2017

COPYRIGHT (C 2018 by the Society of Nuclear Medicine and Molecular Imaging. are required to achieve a therapeutic effect for children with tumors that are less radiosensitive or not amenable to surgical bulk reduction or in the context of minimal residual disease (2-4). Consequently, normal tissue undergoing growth and development is exposed to and damaged by the effect of off-target radiation, often with sequelae that impair the health of an affected individual for a lifetime $(2,4)$. For this reason, external-beam radiotherapy is not feasible in children with widely metastatic cancer and is used only to treat primary or particularly bulky metastatic tumors.

For these difficult cases, targeted molecular radiotherapy using systemic administration of radionuclides, such as the $\beta$-emitting ${ }^{131} \mathrm{I}$, may be effectively applied to deliver sufficient cytotoxic radioactivity to cancer cells (5). Because of the relatively short range in soft tissues of the radiation emitted during decay, $\beta$-emitters ensure a large energy deposition in small volumes of tissue on a cellular and multicellular scale. One such example of a $\beta$-emitter-radiolabeled targeting moiety that has been successfully used in pediatric radiotherapy for several decades is metaiodobenzylguanidine (MIBG), a sympathomimetic guanethidine derivative (6). Both as monotherapy (often in the palliative setting) and in combination with chemotherapy and autologous stem cell transplantation in curative approaches, systemic administration of ${ }^{131} \mathrm{I}-\mathrm{MIBG}$ is an effective therapy in neuroblastoma, with response rates of up to $46 \%$ (7). However, ${ }^{131}$ I-MIBG therapy is not possible in approximately $10 \%$ of neuroblastoma tumors because they do not incorporate MIBG. Other devastating pediatric solid cancers, such as rhabdomyosarcoma, Ewing sarcoma, and osteosarcoma, do not express a widely targetable marker for molecular radiotherapy. Hence, even with standard multimodality therapy, most pediatric patients with metastatic or relapsed solid tumors face a poor prognosis. The chance of cure for high-risk neuroblastoma is approximately $50 \%$, and for children with primary or relapsed metastatic rhabdomyosarcoma it is less than $25 \%$; patients with recurrent Ewing sarcoma have an overall survival of less than $10 \%(8-10)$. These statistics underscore the need for new therapeutic agents, preferably with cancer-specific uptake and broad applicability for these particularly hard-to-cure malignancies.

CLR1404, 18-( $p$-iodophenyl)octadecyl phosphocholine, is an iodinated phospholipid ether analog with selective sequestration in cancer cells. CLR1404 enters cells preferentially via lipid rafts, which are overexpressed in the plasma membrane of cancer cells relative to normal cells, leading to remarkable tumor selectivity (11). CLR1404 uptake has been confirmed in more than 60 adult cancer cell lines, xenograft models, and patient-derived tissues, including cancer stem cells, with little uptake in normal cells 
$(11,12)$. CLR1404 has entered clinical trials in adults as a PET/CT imaging agent (labeled with ${ }^{124} \mathrm{I}$ ) and for radiotherapy (labeled with ${ }^{131}$ I; physical properties are detailed in Supplemental Table 1, available at http://jnm.snmjournals.org) $(13,14)$. In addition, we have recently reported the potential of CLR1404 to specifically target and exert an anticancer effect against pediatric neuroblastoma both in vitro and in vivo in murine xenograft models (12), with no detectable hematotoxicity or adverse effects on the animal health status. The favorable toxicologic profile of CLR 1404 after parenteral administration $(12,15)$ and its broad cancer-targeting ability (11) recommend CLR1404 as a carrier for ${ }^{131}$ I radiotherapy in disseminated disease.

Many critical differences exist between pediatric and adult solid tumors. Although most pediatric cancers have an embryonal origin, that is, are derived from early-differentiating, fetoembryonal tissues, most adult solid tumors are instead derived from mature epithelial tissues (16). Genetic analyses of pediatric cancers have shown many differences from adult cancers, making pediatric cancers distinct diseases (17) that require different treatment strategies with completely different outcomes from adult cancers. In this study, we tested the hypothesis that the preferential uptake of CLR1404 by cancer cells may be exploited for molecularly targeted radiotherapy of pediatric solid tumors, in vivo, using xenograft models of neuroblastoma, rhabdomyosarcoma, Ewing sarcoma, and osteosarcoma.

\section{MATERIALS AND METHODS}

\section{Cells}

We cultured as previously described $(12,18)$ the alveolar rhabdomyosarcoma cell lines Rh30 and Rh41 and the Ewing sarcoma cell lines TC-71 and TC-106 (courtesy of the Children's Oncology Group Cell Repository), the neuroblastoma cell lines CHLA-20 and NB-1691 (courtesy of Dr. Andrew Davidoff, St. Jude Children's Research Hospital), the osteosarcoma cell line SAOS-2 and the embryonal rhabdomyosarcoma cell line RD (ATCC), and primary cultures of normal human fibroblasts (courtesy of Dr. Victoria Browning, University of Wisconsin-Madison).

\section{CLR1404 Synthesis and Radioiodination}

18-( $p$-iodophenyl)octadecyl phosphocholine (known as NM404 or CLR1404), its radioactive analogs ${ }^{124}$ I-CLR1404 and ${ }^{131}$ I-CLR1404 (carrying ${ }^{124} \mathrm{I}$ or ${ }^{131} \mathrm{I}$, respectively, instead of the stable ${ }^{127} \mathrm{I}$ ), and fluorescently labeled CLR1404-BODIPY (where ${ }^{127}$ I was replaced with the fluorescent tag BODIPY-FL; Life Technologies) were kindly provided by Cellectar Biosciences. Clinical-grade CLR1404 was radiolabeled with ${ }^{124} \mathrm{I}$ or ${ }^{131} \mathrm{I}$ (Supplemental Table 1) using an isotope-exchange reaction with sodium iodide as published previously (11). Isolated radiochemical yields for radioiodinated ${ }^{131}$ I-CLR1404 prepared under current good-manufacturing-practice guidelines were consistently above $70 \%$, and radiochemical purity exceeded $96 \%$ in all cases. The mean specific activity was $18.5 \times 10^{8} \mathrm{MBq} / \mathrm{mol}$ (range, $7.4-25.9 \times 10^{8} \mathrm{MBq} / \mathrm{mol}$ ).

\section{In Vitro Uptake of CLR1404-BODIPY}

The green fluorescence of cells $\left(5 \times 10^{5}\right.$ cells $\left./ \mathrm{mL}\right)$ after culture for $18 \mathrm{~h}$ with $5 \mu \mathrm{M}$ fluorescent derivative CLR1404-BODIPY (11) was assessed by flow cytometry (MACSQuant Analyzer; Miltenyi Biotec) and analyzed using FlowJo, version 9.2 (FlowJo LLC). The mean fluorescence intensity was corrected for differences in cell size by normalizing to autofluorescence.

\section{Xenograft Models}

Animal care and use were in compliance with institutional review board-approved protocols and National Institutes of Health guidelines. Human tumor xenografts were established in NOD.Cg-Prkdc ${ }^{\text {scid }}$

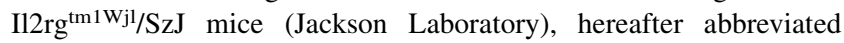
NSG, by inoculating $2 \times 10^{6}$ tumor cells in $200 \mu \mathrm{L}$ of a $3 \mathrm{mg} / \mathrm{mL}$ concentration of Matrigel (BD Biosciences) subcutaneously in the flank. Radioiodinated CLR1404 was administered intravenously when average tumor volumes reached approximately $150 \mathrm{~mm}^{3}$. Injected doses were calculated from the difference in the counts of syringes before and after injection. The animals were housed individually in cages shielded by lead sheets to avoid cumulative irradiation.

\section{PET/CT Imaging and Dosimetry with ${ }^{124}$ I-CLR1404}

Mice were administered, on average, $371 \mathrm{MBq}$ of ${ }^{124}$ I-CLR1404 per kilogram of body weight (range, 296-500 MBq/kg) intravenously and were scanned on an Inveon small-animal PET/CT system (Siemens) at 1, 24,48 , and $72 \mathrm{~h}$ after injection, with additional scans at 96,144 , or $168 \mathrm{~h}$, while anesthetized under $2 \%$ isoflurane. The energy window for ${ }^{124} \mathrm{I}$ was set at $250-650 \mathrm{keV}$, and each mouse was scanned until 40 million counts had been collected to achieve optimal signal-to-noise characteristics. The counts were binned in 3-dimensional histograms, and the resulting images were reconstructed using ordered-subset expectation maximization followed by a maximum a posteriori algorithm. All data were reconstructed without scatter correction. After the imaging, the PET/CT data were preprocessed, and organ and tumor regions of interest were contoured using the AMIRA software (FEI/Thermo Fisher Scientific). The imaging data was then imported into Monte Carlo-based mouse-specific dosimetry software $(14,19)$, to perform 3-dimensional dose calculations. The CT and the PET images were used in the Monte Carlo simulation to define the geometry and source distribution, respectively. The radioactivity of

TABLE 1

Tumor Growth Rate Analysis Using Linear Mixed Effects Model

\begin{tabular}{|c|c|c|c|c|c|}
\hline \multirow[b]{2}{*}{ Xenograft } & \multirow[b]{2}{*}{ Animals $(n)$ per experiment } & \multirow[b]{2}{*}{ Animals $(n)$ per group } & \multicolumn{2}{|c|}{ Slope log volume* } & \multirow[b]{2}{*}{$P^{\dagger}$} \\
\hline & & & Excipient & 131I-CLR1404 & \\
\hline $\mathrm{Rh} 30$ & 14 & 7 & 0.18 & 0.06 & $<0.0001$ \\
\hline TC-71 & 14 & 7 & 0.14 & 0.08 & $<0.0003$ \\
\hline TC-106 & 14 & 7 & 0.26 & 0.10 & $<0.0001$ \\
\hline CHLA-20 & 12 & 6 & 0.13 & 0.09 & 0.0124 \\
\hline NB-1691 & 18 & 9 & 0.15 & 0.09 & $<0.0001$ \\
\hline SAOS-2 & 14 & 7 & 0.11 & 0.08 & $<0.0001$ \\
\hline
\end{tabular}

*Prediction of tumor growth rate (slope of mixed model function, using logarithmic transformation of tumor volumes).

${ }^{\dagger}$ Comparison between predicted tumor growth rates of excipient vs. drug treatment group. 


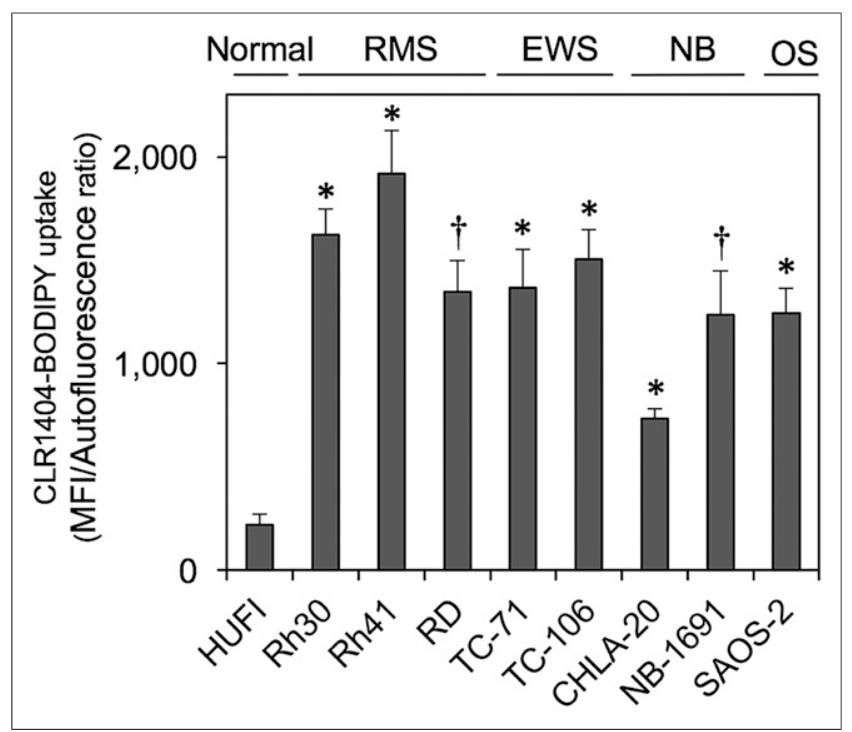

FIGURE 1. Preferential uptake of CLR1404 by pediatric solid cancer cells, compared with normal human fibroblasts (HUFI), by flow cytometry. Data are mean \pm SE from minimum of 3 experiments per cell line. ${ }^{*} P<0.01$. ${ }^{\dagger} P<0.05$. EWS = Ewing sarcoma; MFI = mean fluorescence intensity; $\mathrm{NB}=$ neuroblastoma; OS = osteosarcoma; RMS = rhabdomyosarcoma.

the ${ }^{124}$ I-CLR1404 was converted to the radioactivity of ${ }^{131}$ I-CLR1404 at each time point by assuming the same injection activity and accounting for the difference in physical decay rates. Mean absorbed doses to normal organs and tumor from ${ }^{131}$ I-CLR1404 were estimated for each mouse.

\section{Molecular Radiotherapy with 131|-CLR1404}

Mice bearing xenografts (6-9 animals per group, as detailed in Table 1) were injected with a mean dose $\pm \mathrm{SD}$ of $115 \pm 30 \mathrm{MBq}$ of ${ }^{131} \mathrm{I}-$ CLR1404 per kilogram of body weight (range, $100-180 \mathrm{MBq} / \mathrm{kg}$ ) or an equivalent amount of control nonradioactive CLR1404 (excipient) into the

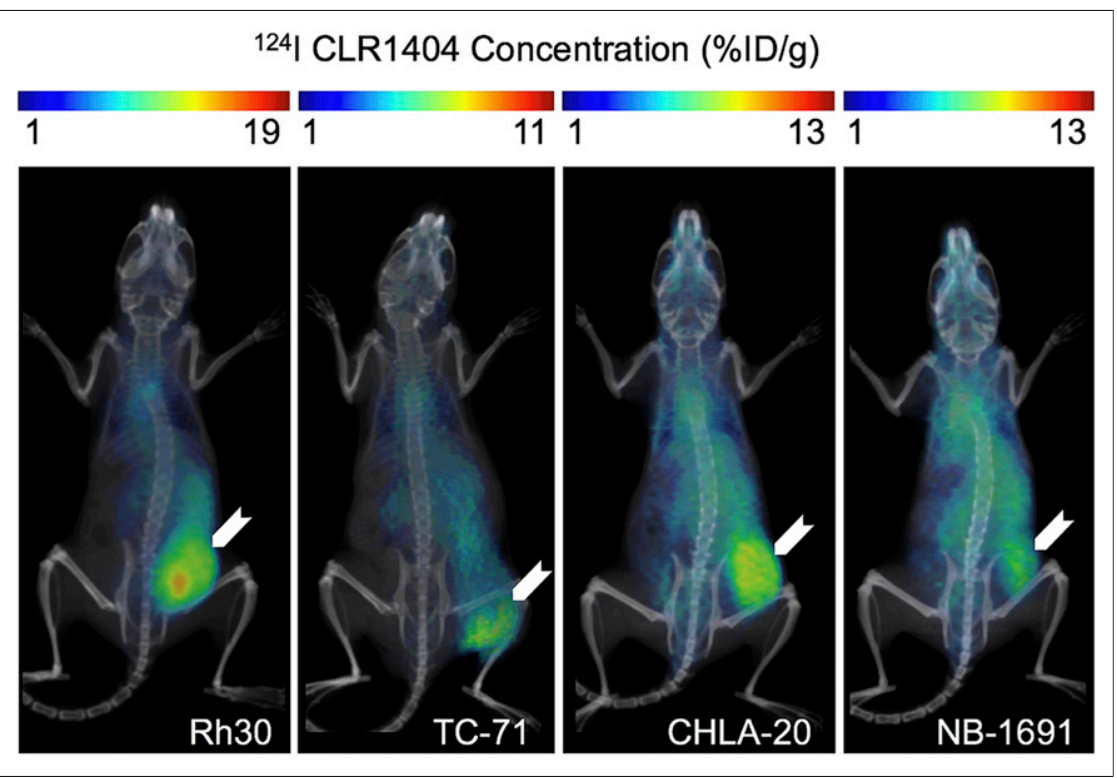

FIGURE 2. Color washes of tissue activity calculated as \%ID/g in xenografts (arrows) and normal tissues of NSG mice $72 \mathrm{~h}$ after ${ }^{124}$ I-CLR1404 administration. Shown are coronal smallanimal PET/CT 3-dimensional volume renderings of 1 representative mouse of 4 scanned for each tumor type. lateral tail vein, and tumor growth was monitored twice weekly. A health score was calculated based on a 5-clinical-criteria evaluation system (modified from Cooke et al. (20)) scoring animal posture, activity, fur texture, weight loss $(<10 \%, 10 \%-20 \%$, or $>20 \%)$, and skin integrity as $0,-1$, or -2 and summing these to a final score of between 0 (normal) and -10 (severe health impairment). To determine treatment-related hematotoxicity in immunocompetent mice, peripheral blood samples from 5 ${ }^{131}$ I-CLR1404-treated BALB/c mice (Jackson Laboratory) (mean treatment dose $\pm \mathrm{SD}, 115 \pm 4 \mathrm{MBq} / \mathrm{kg}$; range, $111-122 \mathrm{MBq} / \mathrm{kg}$ ) were collected before treatment and 2, 4, and $6 \mathrm{wk}$ after treatment and evaluated for platelet and erythrocyte counts, hemoglobin concentration, and leukocyte differential analysis on a VETScan HM5 hematology analyzer (Abaxis).

\section{Statistical Analysis}

Tumor growth was analyzed and compared between the 2 experimental groups by a linear mixed-effects model using SAS software (SAS Institute Inc.), with the group as the fixed effect and the animal as the random effect (to account for repeated measures on the same animals) and the log-transformed tumor volume used as the outcome measure (21). Groups were compared using the $t$ test. Kaplan-Meier survival curves were used to depict the time for tumors to reach or exceed $4,000 \mathrm{~mm}^{3}$, set as the criterion for euthanasia.

\section{RESULTS}

\section{Pediatric Solid Cancer Cells Take Up and Retain CLR1404}

Using green fluorescent BODIPY-labeled CLR1404, we determined its accumulation in various pediatric solid cancer cell lines by flow cytometry. CLR1404-BODIPY was previously shown to display tumor uptake and retention similar to that of the radioiodinated CLR1404 analogs (11). All tested cell lines sequestered and retained significantly higher amounts of CLR1404-BODIPY (average 6.6-fold higher; range, 5.6-7.3) than did human primary normal cell cultures (Fig. 1), corroborating our previously published results (11). Uptake varied between cell lines and cancer types.

\section{I-CLR1404 Targets Pediatric Solid Cancer Cells In Vivo}

In vivo uptake and retention of ${ }^{124} \mathrm{I}$ CLR1404 in tumors was evaluated by small-animal PET/CT in NSG mice bearing human xenografts (4 mice for each tumor cell line: Rh30, TC-71, CHLA-20, and NB-1691). ${ }^{124}$ I-CLR1404 showed preferential accumulation in human cancer cell xenografts $72 \mathrm{~h}$ after intravenous administration (Fig. 2), reaching a percentage injected dose per gram $(\% \mathrm{ID} / \mathrm{g})$ of $7-12$ in Rh-30 rhabdomyosarcoma xenografts, 3-6 in TC-71 Ewing sarcoma, 3-9 in CHLA-20 neuroblastoma tumors, and 4-7 in NB-1691 xenografts. Although ${ }^{124}$ I-CLR1404 was detected in the blood pool at high levels at 1 and $24 \mathrm{~h}$ after injection (evidenced in \% ID/g determined in the heart), it subsequently decreased in normal tissues, paralleling its accumulation in tumors (Supplemental Tables 2-5). In normal tissues, the activity remained mostly detectable in organs with high blood flow, such as the heart, lungs, liver, and spleen, consistent with other small-animal biodistribution studies. 


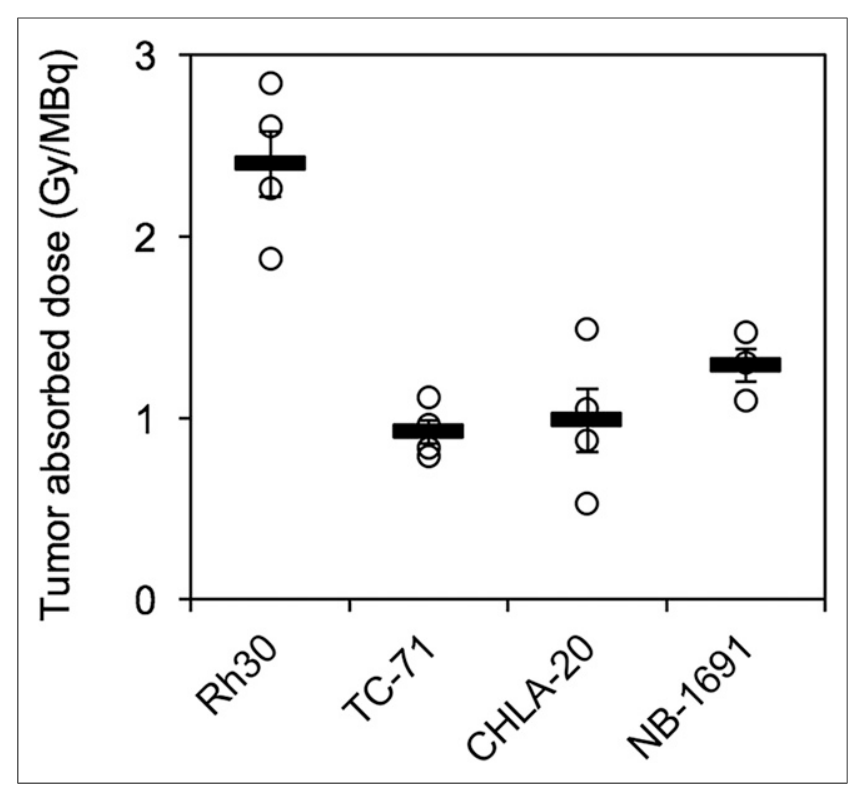

FIGURE 3. Calculated ${ }^{131} \mathrm{I}-\mathrm{CLR} 1404$ absorbed dose per individual mouse xenograft (circles) and mean (thick horizontal bars) \pm SE (thin horizontal bars). Each circle represents 1 mouse $(n=4)$.

\section{Calculated Cumulative Absorbed Dose of ${ }^{131}$ I-CLR1404 Is Highest in Rh30 Xenografts}

The estimated cumulative absorbed doses from ${ }^{131}$ I-CLR1404 were calculated using mouse-specific imaging data (Fig. 3). The largest cumulative absorbed dose delivered to a specific xenograft was in the Rh30 rhabdomyosarcoma xenograft mice, at an average of $2.4 \pm 0.42 \mathrm{~Gy} / \mathrm{MBq}$. Average cumulative absorbed doses of $1.21 \pm 0.53 \mathrm{~Gy} / \mathrm{MBq}$ and $1.10 \pm 0.25 \mathrm{~Gy} / \mathrm{MBq}$ were calculated for the NB-1691 and CHLA-20 xenografts. The lowest cumulative absorbed dose was in the TC-71 Ewing sarcoma tumors, at an average of $0.92 \pm 0.15 \mathrm{~Gy} / \mathrm{MBq}$.

\section{I-CLR1404 Slows Growth of Pediatric Solid Cancer Xenografts and Improves Survival of Xenografted Animals}

To test and exploit the cancer-targeting capacity of this new drug for radiotherapeutic purposes in pediatric cancers, groups of 6-9 xenograft-bearing mice were treated with a single dose of clinical-grade ${ }^{131}$ I-CLR1404. The single-dose treatment significantly slowed growth of all 4 pediatric solid cancer types tested, when compared with control animals treated with excipient containing the equivalent amount of nonradioactive CLR1404 (Fig. 4 and Supplemental Figs. 1-3; Table 1). The evaluation of individual tumor growth patterns indicated that, starting $1 \mathrm{wk}$ after initiation of treatment, mice treated with ${ }^{131}$ I-CLR1404 have on average 2- to 3-fold smaller tumors than excipient-treated animals (for Ewing sarcoma, rhabdomyosarcoma, and osteosarcoma xenografts), or at least $1,000 \mathrm{~mm}^{3}$ smaller than excipient-treated animals (for neuroblastoma tumors). The in vivo data support a strong therapeutic effect of ${ }^{131}$ I-CLR1404 over aggressive tumor growth rates during the first 3-4 wk after treatment (corresponding to $\leq 4{ }^{131}$ I halflives). The treatment of xenografted mice with ${ }^{131}$ I-CLR1404 led to an extension of median survival, and this extension was statistically significant for animals xenografted with Ewing sarcoma and rhabdomyosarcoma (Fig. 5 and Supplemental Fig. 4).
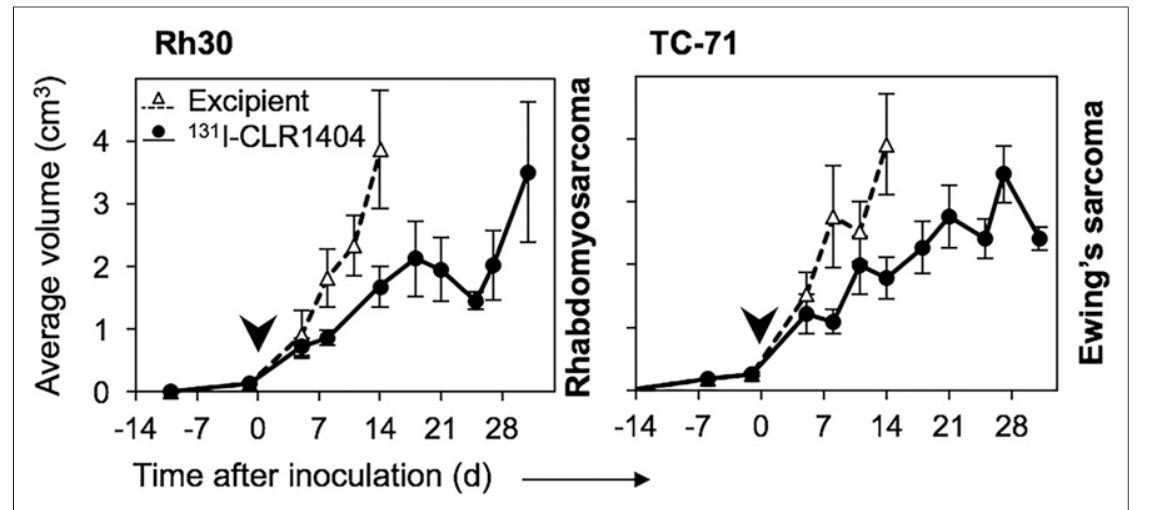

\section{Molecular Radiotherapy Using 131I-CLR1404 Does Not Induce Overt Radiotoxicity}

Radiotoxicity was monitored in all NSG mice bearing sarcoma xenografts. Most of the animals continued to gain weight during the 30- to 60-d observation period (Supplemental Fig. 5A). The animals showed no signs of severe acute or chronic morbidity, with health score minimums averaging -3 (median, -3.5 on a scale of 0 to -10 ) between weeks 2 and 4 after treatment but normalizing afterward (Supplemental Fig. 5B, Ewing sarcoma, rhabdomyosarcoma), except for one animal in the TC-106 Ewing sarcoma group. Weight loss as an indication of systemic radiation toxicity was observed during the first $3 \mathrm{wk}$ of radiotherapy in $65 \%$ of NSG mice (average maximum weight loss across all ${ }^{131}$ I-CLR1404-treated mice, 5.3\%) and was the single most important health criterion decreasing the animal health scores (Supplemental Fig. 5B). In wild-type animals, health scores were zero (unaffected), and average blood counts and hemoglobin concentration were maintained within the reference ranges after treatment with the same doses of ${ }^{131}$ I-CLR1404 as were given
FIGURE 4. Radiotherapeutic effect of ${ }^{131} \mathrm{I}$-CLR1404 in pediatric solid-cancer-xenograft NSG models vs. excipient. Shown are mean \pm SE tumor volumes for duration of $4^{131}$ I half-lives. Arrowhead $=$ start of treatment. Number of animals is detailed in Table 1. 


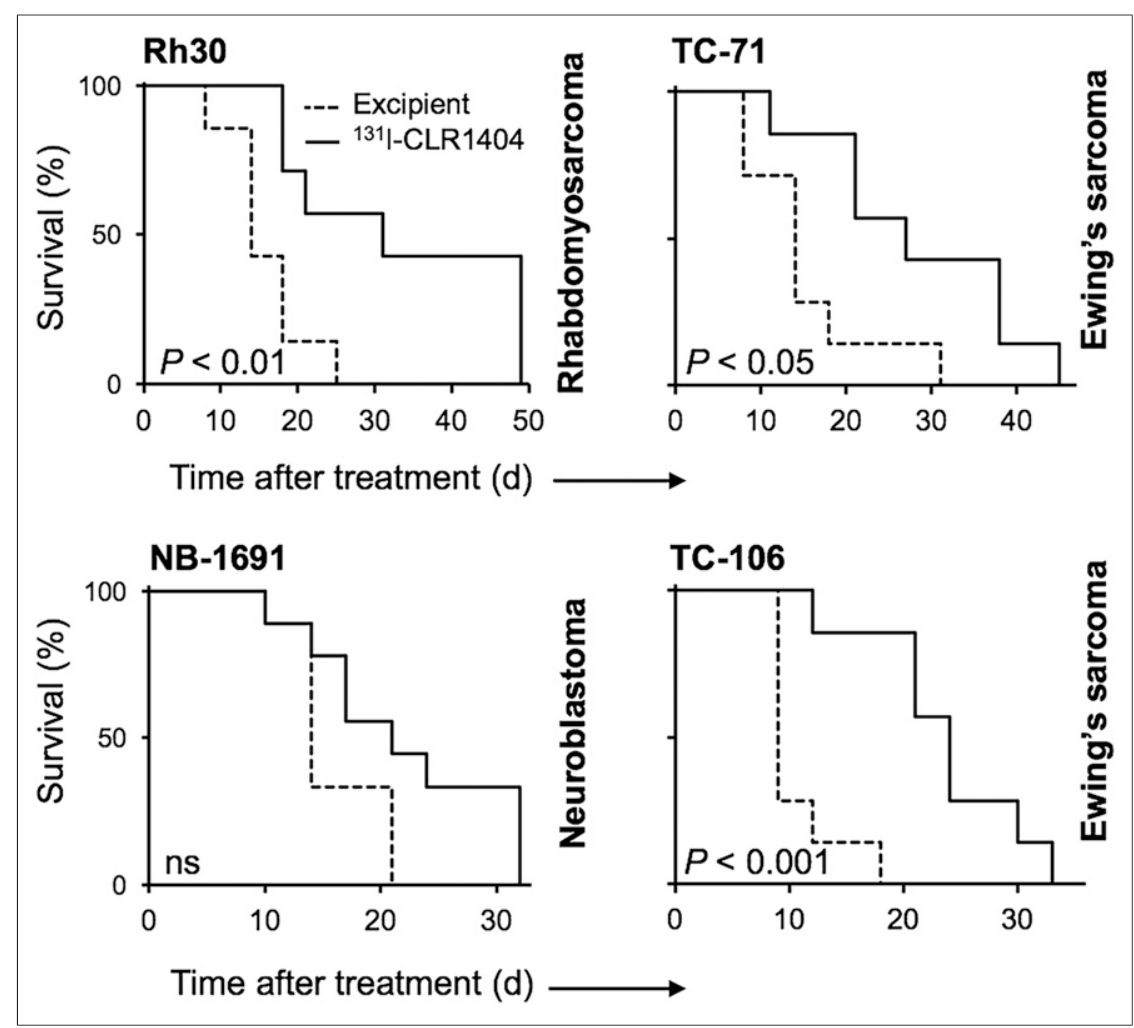

FIGURE 5. Kaplan-Meier survival analysis in pediatric xenograft models after administration of ${ }^{131}$ I-CLR1404. ns $=$ not significant. Number of animals is detailed in Table 1.

wider array of cancer types and tumors of practically every tissue origin, including neuroblastoma, rhabdomyosarcoma, Ewing sarcoma, and osteosarcoma, a characteristic that emphasizes its broad applicability in targeting pediatric solid cancers. Broad applicability is paramount in pediatric oncology from a drug development and regulatory standpoint, given the rarity of cases per tumor type, patient variability in targetable markers, and recoverable costs for preclinical and clinical development. The last of these is a major barrier for the pediatricsspecific development of anticancer treatments by the pharmaceutical industry, reflected by the fact that few drugs have been developed in the last $20 \mathrm{y}$ specifically to treat pediatric cancers (26).

Paralleling the in vitro CLR1404 uptake results, we observed differences in tumorspecific absorbed doses among the 3 cancer types in vivo. The $\% \mathrm{ID} / \mathrm{g}$ of ${ }^{124} \mathrm{I}$ that accumulated in our tumor xenografts using CLR1404 as the carrier was higher than observed with, for instance, ${ }^{124} \mathrm{I}-\mathrm{MIBG}$. Seo et al. reported ${ }^{124}$ I-MIBG uptake of less than $2 \% \mathrm{ID} / \mathrm{g}$ in mouse subcutaneous human neuroblastoma xenografts overexpressing human norepinephrine transporter. In their study, the $\% \mathrm{ID} / \mathrm{g}$ of ${ }^{124} \mathrm{I}$ was higher

to the tumor-bearing animals (Supplemental Figs. 5C and 5D), suggesting an acceptable therapeutic index for ${ }^{131}$ I-CLR1404.

\section{DISCUSSION}

Our results indicate that CLR1404 is an efficient cancertargeting molecule in pediatric solid cancers, with minor uptake in normal tissues. The preferential uptake of CLR1404 and its radioactive analogs in cancer cells is extremely important for both diagnostic imaging and radiotherapy in these difficult-to-cure pediatric malignancies, particularly in the setting of primary disseminated disease or relapse. Few radiolabeled compounds are available in pediatric oncology tumor detection and therapy, and most are used for diagnostic imaging. ${ }^{18} \mathrm{~F}-\mathrm{FDG}$ PET, in combination with ${ }^{18} \mathrm{~F}$-FDG PET/CT, has advanced as the most widely used functional diagnostic imaging tool for a broad variety of pediatric solid tumors (22). However, high glucose-consumption by brain or inflammatory cells results in ${ }^{18}$ F-FDG uptake and can obscure tumor tissue or lead to false-positive results, an important limitation of this imaging modality (22). ${ }^{123}$ I-MIBG scintigraphy is routinely prescribed for initial diagnosis, staging, and response assessment in neuroblastoma. Other theranostic radioactive small molecules are being developed and are in clinical testing, such as DOTATATE for neuroendocrine tumors, including neuroblastoma $(23,24) .{ }^{131}$ I-MIBG for the treatment of neuroblastoma is currently perhaps the only widely accepted targeted molecular radiotherapy, available at specialized treatment centers with suitable infrastructure. In osteosarcoma, ${ }^{153} \mathrm{Sm}$-ethylenediamine-tetramethylenephosphonic acid is available for the palliative treatment of bone lesions (25). In stark contrast to these targeted radiotherapeutic molecules, CLR1404 and its radioactive analogs target a much in tumors implanted under the renal capsule but did not exceed on average 3\%-4\% after the first $24 \mathrm{~h}$, with a rapid decline thereafter (27). However, we did not perform a direct comparison between ${ }^{124}$ I-MIBG and ${ }^{124}$ I-CLR1404 in neuroblastoma xenograft models, which limits our ability to compare the 2 carriers.

The selective uptake and retention of ${ }^{131}$ I-CLR1404 in tumors were ascertained by dosimetry, biodistribution, and pharmacokinetic studies in the context of 2 completed clinical trials in adults with advanced solid cancers $(13,28)$. As we have previously shown, ${ }^{124}$ I-CLR1404 is an ideal agent for pretreatment dosimetry in the preclinical and clinical settings $(14,19)$ and thus may allow the calculation and prescription of personalized, patient-specific doses, as is currently routine for external-beam irradiation (29). This is particularly important given the variety of tumors that can potentially be treated with ${ }^{131}$ I-CLR1404 and the expected variability in tumor- or patient-specific absorbed doses and will allow for important optimization of the therapeutic ratio, which is of great concern specifically in the pediatric setting. Such applications are supported by previous observations in the clinical setting indicating that imaging using a $0.02 \mathrm{mg} / \mathrm{kg}$ dose of ${ }^{124} \mathrm{I}-\mathrm{CLR} 1404$ would not prevent therapeutic doses of ${ }^{131}$ I-CLR1404 from entering tumors (30).

The capacity of ${ }^{131}$ I-CLR1404 to deliver effective radiation doses to a variety of pediatric solid cancer xenografts has the potential to soon profoundly affect the clinical management of these patients. Administration of a single dose with a mean activity of $115 \mathrm{MBq} / \mathrm{kg}$, although not curative, demonstrated statistically significant therapeutic activity in all 4 types of pediatric solid tumors tested (Table 1; Fig. 4). Notably, all cell lines that were used to establish xenografts in this study are derived from highly aggressive human tumors that are postchemotherapy multidrug-resistant 
and bear markers of aggressiveness such as MYCN amplification (NB-1691) (31), nonfunctional p53 (TC-71), or chromosomal translocations (Rh30) (32). By estimating the cumulative absorbed doses through quantitative imaging, we determined that doses of $0.5-2.8 \mathrm{~Gy} / \mathrm{MBq}$ were delivered to solid tumor xenografts with our one-time, nonoptimized dose of molecularly targeted radiotherapy. Compared with single-fraction externalbeam radiation therapy, these doses would be considered suboptimal in controlling heterotopic solid tumor xenografts in immunodeficient mouse models. Our study was not geared toward therapy optimization but rather was designed to show the first proof of principle in the preclinical setting. Because of a specific defect in the Prkdc gene, which is involved in DNA damage repair, the NSG mouse strain is markedly radiosensitive but allows the reliable xenotransplantation of a broad variety of human cancer tissues because of the profound immunosuppression (33). This characteristic enabled us to establish an array of pediatric cancer xenograft models, but we chose to limit the injected radioactivity to avoid excessive tissue radiotoxicity while accepting decreased therapeutic effects. We have previously published data on adult tumor xenograft models in other immunodeficient mouse strains, in which single ${ }^{131}$ I-CLR1404 doses of up to $200 \mathrm{MBq} / \mathrm{kg}$ were administered, leading to significant tumor shrinkage and even cure of some animals with certain cancer types (11).

In this context, the recent results of a phase I multicenter openlabel pilot study on 10 adult patients with relapsed or refractory advanced solid malignancies are encouraging, showing disease stabilization in $40 \%$ of the patients receiving ${ }^{131}$ I-CLR1404 therapy $(13,28)$ at the maximum dose administered, $1,387 \mathrm{MBq} / \mathrm{m}^{2}$ $(\sim 30 \mathrm{MBq} / \mathrm{kg})$. This dose is considerably lower than prescribed in the context of ${ }^{131} \mathrm{I}-\mathrm{MIBG}$ therapy for children with neuroblastoma (on average, 450-550 MBq/kg), in which transient thrombocytopenia or neutropenia are expected and are alleviated by platelet transfusion or growth factor treatment (e.g., with filgrastim) or, for higher radioactive doses, autologous stem cell support (6). However, because the pharmacokinetic properties of CLR1404 $(13,34)$ differ from those of MIBG (35), only a clinical study can clarify the maximum tolerated dose of this compound in children. We therefore believe that in a clinical trial with appropriate supportive care, higher doses of ${ }^{131}$ I-CLR1404 could be tolerated than we have tested in our preclinical mouse model and ultimately might improve the antitumor effects.

\section{CONCLUSION}

Our data indicate that ${ }^{131} \mathrm{I}$-CLR1404 is an effective and welltolerated molecularly targeted radiotherapeutic agent in mouse models of high-risk pediatric solid cancers that have a particularly dismal outcome in patients with relapsed, refractory, or disseminated disease. Although a pediatric clinical trial is in development at our institution, future preclinical research will be geared toward integrating ${ }^{131}$ I-CLR1404 in multimodality therapy approaches, in combination with chemotherapy, external-beam radiotherapy, or immunotherapy.

\section{DISCLOSURE}

This work was supported by grant R21CA198392-01 from NIH, grant P30CA014520 from NIH/NCI, the Midwest Athletes against Childhood Cancer Foundation, Hyundai Hope on Wheels, and translational research grant SU2C-AACR-DT1113 from Stand Up to Cancer/St. Baldrick's Pediatric Dream Team. Jamey P. Weichert was a cofounder of Cellectar Biosciences. No other potential conflict of interest relevant to this article was reported.

\section{REFERENCES}

1. Wolden SL, Anderson JR, Crist WM, et al. Indications for radiotherapy and chemotherapy after complete resection in rhabdomyosarcoma: a report from the intergroup rhabdomyosarcoma studies I to III. J Clin Oncol. 1999;17: 3468-3475.

2. Laverdiére C, Cheung NK, Kushner BH, et al. Long-term complications in survivors of advanced stage neuroblastoma. Pediatr Blood Cancer. 2005;45: 324-332.

3. Indelicato DJ, Keole SR, Shahlaee AH, Shi W, Morris CG, Marcus RB Jr. Definitive radiotherapy for Ewing tumors of extremities and pelvis: long-term disease control, limb function, and treatment toxicity. Int J Radiat Oncol Biol Phys. 2008;72:871-877.

4. Spix C, Pastore G, Sankila R, Stiller CA, Steliarova-Foucher E. Neuroblastoma incidence and survival in European children (1978-1997): report from the Automated Childhood Cancer Information System Project. Eur J Cancer. 2006;42: 2081-2091.

5. Schmidt M, Baum RP, Simon T, Howman-Giles R. Therapeutic nuclear medicine in pediatric malignancy. $Q \mathrm{~J} \mathrm{Nucl} \mathrm{Med} \mathrm{Mol} \mathrm{Imaging.} \mathrm{2010;54:411-428.}$

6. Parisi MT, Eslamy H, Park JR, Shulkin BL, Yanik GA. ${ }^{131}$ I-metaiodobenzylguanidine theranostics in neuroblastoma: historical perspectives; practical applications. Semin Nucl Med. 2016;46:184-202.

7. Polishchuk AL, DuBois SG, Haas-Kogan D, Hawkins R, Matthay KK. Response, survival, and toxicity after iodine-131-metaiodobenzylguanidine therapy for neuroblastoma in preadolescents, adolescents, and adults. Cancer. 2011;117: 4286-4293.

8. Lager JJ, Lyden ER, Anderson JR, Pappo AS, Meyer WH, Breitfeld PP. Pooled analysis of phase II window studies in children with contemporary high-risk metastatic rhabdomyosarcoma: a report from the Soft Tissue Sarcoma Committee of the Children's Oncology Group. J Clin Oncol. 2006;24: 3415-3422.

9. Bacci G, Ferrari S, Longhi A, et al. Therapy and survival after recurrence of Ewing's tumors: the Rizzoli experience in 195 patients treated with adjuvant and neoadjuvant chemotherapy from 1979 to 1997. Ann Oncol. 2003;14:16541659.

10. Park JR, Bagatell R, London WB, et al. Children's Oncology Group's 2013 blueprint for research: neuroblastoma. Pediatr Blood Cancer. 2013;60:985993.

11. Weichert JP, Clark PA, Kandela IK, et al. Alkylphosphocholine analogs for broad-spectrum cancer imaging and therapy. Sci Transl Med. 2014;6:240ra75.

12. Marino R, Baiu DC, Bhattacharya S, et al. Tumor-selective anti-cancer effects of the synthetic alkyl phosphocholine analog CLR1404 in neuroblastoma. Am J Cancer Res. 2015;5:3422-3435.

13. Grudzinski JJ, Titz B, Kozak K, et al. A phase 1 study of ${ }^{131}$ I-CLR1404 in patients with relapsed or refractory advanced solid tumors: dosimetry, biodistribution, pharmacokinetics, and safety. PLoS One. 2014;9:e111652.

14. Besemer A, Grudzinski J, Titz B, Bednarz JB. Evaluation of dosimetric uncertainties in individualized targeted radionuclide therapy (TRT) treatment planning using pre-clinical data [abstract]. Med Phys. 2015;42:3675.

15. Jiang H, Cannon MJ, Banach M, et al. Quantification of CLR1401, a novel alkylphosphocholine anticancer agent, in rat plasma by hydrophilic interaction liquid chromatography-tandem mass spectrometric detection. J Chromatogr B Analyt Technol Biomed Life Sci. 2010;878:1513-1518.

16. Simone JV, Lyons J. The evolution of cancer care for children and adults. J Clin Oncol. 1998;16:2904-2905.

17. Downing JR, Wilson RK, Zhang J, et al. The Pediatric Cancer Genome Project. Nat Genet. 2012;44:619-622.

18. Kang MH, Smith MA, Morton CL, Keshelava N, Houghton PJ, Reynolds CP. National Cancer Institute pediatric preclinical testing program: model description for in vitro cytotoxicity testing. Pediatr Blood Cancer. 2011;56: 239-249.

19. Morris ZS, Weichert JP, Saker J, et al. Therapeutic combination of radiolabeled CLR1404 with external beam radiation in head and neck cancer model systems. Radiother Oncol. 2015;116:504-509.

20. Cooke KR, Kobzik L, Martin TR, et al. An experimental model of idiopathic pneumonia syndrome after bone marrow transplantation: $\mathrm{I}$. the roles of minor $\mathrm{H}$ antigens and endotoxin. Blood. 1996;88:3230-3239.

21. Laird NM, Ware JH. Random-effects models for longitudinal data. Biometrics. 1982;38:963-974. 
22. Uslu L, Donig J, Link M, Rosenberg J, Quon A, Daldrup-Link HE. Value of ${ }^{18} \mathrm{~F}-$ FDG PET and PET/CT for evaluation of pediatric malignancies. J Nucl Med. 2015;56:274-286.

23. Kong G, Hofman MS, Murray WK, et al. Initial experience with gallium- 68 DOTA-octreotate PET/CT and peptide receptor radionuclide therapy for pediatric patients with refractory metastatic neuroblastoma. J Pediatr Hematol Oncol. 2016;38:87-96.

24. Gains JE, Bomanji JB, Fersht NL, et al. ${ }^{177}$ Lu-DOTATATE molecular radiotherapy for childhood neuroblastoma. J Nucl Med. 2011;52:1041-1047.

25. Anderson $P$, Nunez R. Samarium lexidronam ( ${ }^{153} \mathrm{Sm}$-EDTMP): skeletal radiation for osteoblastic bone metastases and osteosarcoma. Expert Rev Anticancer Ther. 2007; 7:1517-1527.

26. Adamson PC. Improving the outcome for children with cancer: development of targeted new agents. CA Cancer J Clin. 2015;65:212-220.

27. Seo Y, Gustafson WC, Dannoon SF, et al. Tumor dosimetry using $\left[{ }^{124} \mathrm{I}\right] \mathrm{m}$ iodobenzylguanidine microPET/CT for $\left[{ }^{131} \mathrm{I}\right] \mathrm{m}$-iodobenzylguanidine treatment of neuroblastoma in a murine xenograft model. Mol Imaging Biol. 2012;14:735-742.

28. Lubner SJ, Mullvain J, Perlman S, et al. A phase 1, multi-center, open-label, dose-escalation study of ${ }^{131}$ I-CLR1404 in subjects with relapsed or refractory advanced solid malignancies. Cancer Invest. 2015;33:483-489.
29. McGowan DR, Guy MJ. Time to demand dosimetry for molecular radiotherapy? Br J Radiol. 2015;88:20140720-20140723.

30. Grudzinski JJ, Burnette RR, Weichert JP, Jeraj R. Dosimetric effectiveness of targeted radionuclide therapy based on a pharmacokinetic landscape. Cancer Biother Radiopharm. 2010;25:417-426.

31. He J, Gu LB, Zhang HL, Zhou MX. Crosstalk between MYCN and MDM2-p53 signal pathways regulates tumor cell growth and apoptosis in neuroblastoma. Cell Cycle. 2011;10:2994-3002.

32. Rodriguez-Perales S, Martinez-Ramirez A, de Andres SA, et al. Molecular cytogenetic characterization of rhabdomyosarcoma cell lines. Cancer Genet Cytogenet. 2004;148:35-43.

33. Shultz LD, Banuelos S, Lyons B, et al. NOD/LtSz-Rag1nullPfpnull mice: a new model system with increased levels of human peripheral leukocyte and hematopoietic stem-cell engraftment. Transplantation. 2003;76:10361042 .

34. Grudzinski JJ, Floberg JM, Mudd SR, et al. Application of a whole-body pharmacokinetic model for targeted radionuclide therapy to NM404 and FLT. Phys Med Biol. 2012;57:1641-1657.

35. Ehninger G, Klingebiel T, Kumbier I, et al. Stability and pharmacokinetics of $\mathrm{m}-\left[{ }^{131} \mathrm{I}\right]$ iodobenzylguanidine in patients. Cancer Res. 1987;47:6147-6149. 